éphémérides pour l'année r950, le centre publie le volume annuel d'éphémérides sous les auspices de l'U.A.I.

II. La Commission recommande qu'une subvention annuelle de mille dollars soit donnée au centre des Petites Planètes pour la publication de ces circulaires et éphémérides.

I2. La Commission recommande que le centre des Petites Planètes à Cincinnati soit autorisé à attribuer des désignations provisoires et des numéros définitifs aux Petites Planètes, et à approuver et confirmer les noms proposés pour elles par les auteurs de leur découverte.

r3. La Commission attire l'attention sur le manque d'observations des comètes faibles par les observatoires de l'hémisphère sud et recommande que des mesures soient prises par les observatoires convenablement équipés, pour obtenir spécialement dans le cas des comètes périodiques, au moins les observations essentielles de positions et de magnitude, lorsque les comètes ne sont pas observables dans l'hémisphère nord. Elle recommande que ces observations soient communiquées sans délai au Bureau de l'U.A.I.

I4. La Commission émet le vœu que soit poursuivie la recherche entreprise à l'Observatoire de Nice, en vue d'identifier les astéroïdes découverts depuis Igoo et dont on n'a pu obtenir que deux positions.

I5. La Commission exprime de nouveau un vif intérêt pour les Research Surveys of Minor Planets, préparés et publiés sous la direction de son président d'honneur A. O. Leuschner, et recommande qu'ils soient continués. Elle exprime le vœu que les moyens puissent être trouvés pour la réalisation de cette œuvre.

\title{
Commission 22. (MÉTÉoREs)
}

I. La Commission recommande que des observations de météores faites simultanément par les méthodes électroniques (radar) et visuelles ou photographiques soient étendues, parce que cette commission est convaincue que la continuation et l'extension des observations électroniques (radar) sont actuellement essentielles pour le progrès de l'astronomie relative aux météores et les recherches qui s'y rattachent.

2. La Commission recommande que des cartes stellaires destinées aux observations visuelles de météores soient préparées, et qu'une sous-commission soit constituée pour étudier la forme de ces cartes et les modalités de leur publication; cette commission sera présidée par M. Millman et elle comprendra MM. Bečvář, Olivier et Prentice.

3. Que les astronomes appellent l'attention des physiciens sur l'application des méthodes nouvelles de recherche à l'étude des météorites faite dans les laboratoires; de telles recherches doivent embrasser d'une façon systématique toutes les classes représentatives de météorites.

\section{Commission 22. (MÉTÉoRES)}

\section{Sous-commission de la lumière zodiacale et de la lumière du ciel nocturne}

I. En vue de la recherche d'une influence possible de matériaux extra-terrestres sur l'ionisation anormale de l'ionosphère, il y a lieu d'effectuer des mesures aussi précises que possible des variations saisonnières et autres de la brillance de la lumière zodiacale.

(Résolution transmise par la commission mixte de l'ionosphère du Conseil International des Unions Scientifiques.)

2. Pour permettre la compréhension des processus atomiques dans l'ionosphère, il est nécessaire d'obtenir des spectres des aurores polaires et du ciel nocturne avec une meilleure définition et une plus grande dispersion que ceux obtenus jusqu'à présent.

(Résolution transmise par la commission mixte de l'ionosphère du Conseil International des Unions Scientifiques.) 
3. La sous-commission, prenant en considération le plan de travail étendu, suggéré par le Dr Whipple, estime qu'elle doit être tenue au courant de tous les progrès réalisés dans l'étude de la haute atmosphère, quelles que soient les méthodes utilisées.

Elle souhaite qu'à cet effet une liaison étroite soit établie entre elle et les commissions de l'Union Géophysique Internationale et de l'U.R.S.I., s'occupant de problèmes connexes.

\section{Commission 23. (CARTE DU CIEL)}

I. L'Assemblée Générale de l'U.A.I. et la Commission de la Carte du Ciel attirent l'attention des Gouvernements du Commonwealth et des Etats Australiens sur l'importance qui s'attache à l'achèvement des zones du Catalogue Photographique du Ciel confiées aux observatoires australiens; elles leur demandent d'en favoriser l'exécution en programme d'urgence.

Les dernières lacunes du Catalogue Photographique nuisent à l'utilisation globale de cet instrument scientifique précieux, qui a demandé tant d'efforts internationaux; elles sont d'autant plus regrettables que l'essentiel du travail a déjà été fourni. L'U.A.I. serait reconnaissante à Monsieur H. W. Wood, Government Astronomer, Sydney, New South Wales, d'accepter la direction de cet achèvement et à Monsieur le Dr R. v. d. R. Woolley, Directeur de l'Observatoire du Commonwealth, Canberra, de lui fournir les aides nécessaires.

La Commission de la Carte du Ciel s'engage à demander l'aide financière de l'U.A.I. pour la publication des derniers volumes, lorsque les manuscrits seront prêts pour l'impression.

2. La Commission de la Carte du Ciel, désireuse de voir se terminer la publication du Catalogue Photographique, demande à l'U.A.I. de mettre à sa disposition, en plus des fonds qui lui ont été attribués et qui ne sont pas encore dépensés, une somme de vingt-et-un mille francs-or pour couvrir les frais d'impression de 5 volumes du Catalogue, appartenant aux zones laissées en souffrance par les Observatoires de Potsdam, de Perth et de Rio-deJaneiro et dont les manuscrits, préparés par les Observatoires d'Oxford et d'Edimbourg, pourraient être imprimés d'ici I95I.

\section{Commission 24. (PARAllaXes Stellaires et Mouvements PROPRES)}

La Commission recommande que le Comité Exécutif soit prié d'allouer un montant de \$1500 pour les frais d'impression et de reliure de la troisième édition du General Catalogue of Parallaxes. L'Observatoire de Yale a entrepris la compilation et la préparation du manuscrit dactylographié pour l'impression.

\section{Commission 27. (ÉTOILES VARIABLES)}

I. La Commission recommande que de petites cartes soient publiées pour chaque étoile variable nouvellement découverte, quand cette étoile ne figure dans aucun catalogue méridien.

2. La Commission recommande qu'une subvention de $\$ 250$ soit mise à la disposition de son président pour rétribuer un dessinateur qui serait chargé de préparer des cartes d'étoiles variables australes pour les astronomes amateurs.

3. La Commission tient à mettre en relief l'importance des observations visuelles de certaines classes d'étoiles variables.

4. La Commission approuve les trois résolutions proposées par MM. H. N. Russell, J. E. Merrill et N. L. Pierce:

$I^{\circ}$. Les époques des minima des variables à éclipses doivent être publiées individuellement, en Temps Moyen de Greenwich, c'est-à-dire, en faisant commencer le jour à midi moyen de Greenwich. Ces époques seront héliocentriques, et exprimées en jours et fractions décimales du jour. En aucun cas, la publication ne pourra être limitée à celle d'une époque normale. Si l'on veut donner les résidus des observations par rapport à une 